\title{
Habilidades sociales en Engagement y desempeño académico en estudiantes universitarios
}

\section{Social skills in Engagement and academic performance in university students}

\author{
Verónica Cajas Bravo ${ }^{1, a}$ \\ https://orcid.org/0000-0001-8939-3733
}

Marco Antonio Paredes Perez ${ }^{2, b}$

https://orcid.org/0000-0001-5963-839X

\section{Lorenzo Pasquel Loarte ${ }^{1, c}$}

https://orcid.org/0000-0002-7740-2329

\author{
Alexander Frank Pasquel Cajas ${ }^{3, d}$ \\ https://orcid.org/0000-0002-0603-0329 \\ ${ }^{1}$ Universidad Nacional Hermilio Valdizán. \\ ${ }^{2}$ Universidad Nacional del Centro. \\ ${ }^{3}$ Pontificia Universidad Católica del Perú. \\ averonica.cajas64@gmail.com \\ b marcoparedes3973@gmail.com \\ c 1 orenzo_pasquel_loarte@hotmail.com \\ dapasquel@pucp.edu.pe
}

Recibido e1: $14 / 09 / 2019$
Aceptado e1: $10 / 01 / 2020$

\begin{abstract}
The study of engagement has become one of the main topics of research in academic contexts. The present investigation sought to determine the relationship between engagement and academic performance in university students. The sample population consisted of 298 students from the National University of Central Peru; the instrument used was the UWES scale on academic engagement; to evaluate performance, consolidated averages were used in three professional careers. The results indicate that there is no significant correlation between the variables studied, the correlation being significant at the 0.05 level (bilateral), with a p-value of 0.33 . The Spearman correlation shows a non-significant relationship level, with a coefficient of 0.123 . Students perform with levels of personal internal motivation; the main conclusion is that despite the adverse conditions and lack of motivation, they maintain a balanced academic performance.
\end{abstract}

Keywords: Social skills, engagement, academic performance.

Palabras clave: Habilidades sociales, engagement, desempeño académico. 


\section{INTRODUCCIÓN}

El hombre es un ser social, la comunicación es un proceso necesario porque permite interactuar, sobrevivir, crecer y aprender de puntos de vista diferentes para vivir en armonía, compartiendo experiencias, fomentando la cohesión social. Relacionarse y desarrollar habilidades sociales es imprescindible en el contexto actual para llegar a acuerdos, evitando conflictos en las relaciones interpersonales. El desarrollo social arranca en los primeros años (Navarro, 2003). La comunicación es una parte esencial de esa vida. Cuando se expresa una necesidad se ponen a prueba las habilidades sociales, las cuales ayudan o entorpecen. Si se logra evaluar cómo está la comunicación y si entrena las herramientas de interacción, se logrará estar mejor, ser más influyentes y tener una mayor facilidad para lograr los propósitos.

El ser humano es social y colaborativo. La forma que tienen de desenvolverse es aprendida y, por tanto, modificable y entrenable. Puede variar con los años por influencias externas o hechos que se viven, y pueden aprenderse determinadas herramientas que se convierten como parte del suyo (Rull, 2019). Algunas carecen de importancia y otras son importantes. Las habilidades sociales, por tanto, se relacionan de forma directa con el bienestar y ayudará a estar cada vez mejor.

La vida afectiva y social representan dimensiones alejadas de los entornos académicos y la educación formal (Riquelme y Munita, 2013). El estudiante necesita desarrollarse en ambientes adecuados y seguros, con calidad de vida que favorezcan su desarrollo personal. Las relaciones interpersonales generadas en sus diversos contextos y en su ámbito escolar son agentes fortalecedores de habilidades sociales (Guadalupe, 2015). Muchos estudiantes en sus relaciones proyectan una identidad ficticia para complacer a otros (De Onzoño, 2016). Las habilidades sociales maximizan la probabilidad de conseguir refuerzo externo (Gismero, 2010). Permiten a los estudiantes mayor capacidad para discernir sobre su comportamiento, controlando sus emociones para expresarlas de manera apropiada, generando así mejor calidad en su vida (García, 2005).

$\mathrm{Ng}$, Bartlett y Elliott (2018) enfatizan que las habilidades sociales son esenciales en el desarrollo y mantenimiento de relaciones exitosas con compañeros, padres, maestros, empleadores y nuevos conocidos. No obstante, el currículo de estudios universitarios no parece manifestar interés en el entrenamiento de habilidades sociales en engagement en los futuros profesionales (Herrera, et al., 2012) aun cuando está demostrada su importancia para esta población (Pades y Roso, 2013; McCarthy. y Trace, 2014). Un alto nivel de habilidades sociales incrementa conductas más saludables (Del Rosal, et al., 2012; Párraga, 2005) ante la crisis social que se agudiza en las sociedades del mundo; por ello, algunas investigaciones muestran preocupación por desarrollar conductas pro-sociales en universitarios (Caballo et al., 2014; Herrera, et al., 2012; León, et al., 2015; Herrera, et al., 2012; Mendo, et al., 2016; Rodríguez, et al., 2014), mediante programas orientados al aprendizaje cooperativo (León, et al., 2016), evaluación de síntomas psicosociales (Wagner, et al., 2014) e intervenciones adaptativas de estudiantes en nuevos escenarios académicos (Chávez, Contreras y Velázquez, 2013).

Engagement es la capacidad humana de experimentar altos niveles de vigor, dedicación y absorción que un estudiante experimenta al realizar sus labores académicas; los estudiantes demuestran inteligencia, motivación y hábitos de estudio en su desempeño académico (Borges, Hernández y González, 2012). Existe considerable evidencia de estudios realizados respecto a los factores que determinan en gran medida el rendimiento académico en estudiantes, los mismos que señalan que una de las variables más importantes es la satisfacción emocional-psicológica y presentan relación con la calificación final obtenida (Guàrdia, et al., 2006). Asimismo, estudios muestran que los alumnos con mayores niveles de engagement son mujeres, desarrollando más actividades al interior de la universidad, al mismo tiempo tenían mejores calificaciones (Parada y Pérez, 2014).

El engagement académico, ha demostrado ser un factor fundamental para impulsar los aprendizajes, rendimiento, interés, disfrute y bienestar psicológico en alumnos. Sin embargo, aún quedan en evidencia ciertas fisuras conceptuales que deben profundizarse y esclarecerse (Medrano, Moretti y Ortiz, 2015). Algunos autores refieren que la definición de este término todavía requiere mucho debate y entendimiento, por ende la descripción detallada de sus características es importante, a fin de nutrir el estado del arte de dicha variable (Compagnucci, Cardós, Denegri, y Barboza, 2002). Las Investigaciones referidas a la asociación entre 
engagement y evaluación del conocimiento en estudiantes deben profundizarse aún más.

Se puede precisar que la variable engagement, es estudiada más dentro del contexto empresarial, por ende, dentro de los círculos académicos su nivel de incidencia es menor, por no haber multiplicidad de estudios en esos entornos. Por ello, la investigación busca establecer la relación existente entre engagement y el desempeño académico en estudiantes de la Universidad Nacional del Centro.

\section{MARCO TEÓRICO}

\section{Habilidades sociales}

Las habilidades sociales involucran procesos de adaptación social con capacidades de empatía, adecuado control de impulsos, trabajo cooperativo, razonamiento, entre otros (Morales, Benites y Agustín, 2013); permiten que las personas puedan adaptarse a diversas situaciones sociales generando pautas cognitivas en esta interacción (Zavala, Valadez, y Vargas, 2008). Es importante mostrar estas habilidades para lograr una adecuada adaptación y convivencia en diversos escenarios (Betancourth, Zambrano, Ceballos, Benavides, y Villota, 2017).

Un aspecto importante es que estas habilidades se aprenden a medida que se establecen interacciones con el entorno; por tanto, pueden ser modificadas y desarrolladas ya que no representan un constructo inamovible en la estructura de personalidad (Rovira, 2018). Investigaciones en neurociencia señalan que activan estructuras cerebrales que permiten una mejor comprensión de los sentimientos del otro, favoreciendo la interacción social (Cacioppo, Cacioppo, y Cole, 2013). Básicamente, buscan enseñarle al individuo cómo llevar a cabo la conducta más beneficiosa para sus intereses sin que salga perjudicado a corto o largo plazo; por ello, mantienen una estrecha relación con las habilidades para la vida, ya que contienen similares competencias a desarrollar (Patricio, Maia, y Bezerra, 2015).

\section{Engagement}

Este término surge de manifestaciones físicas, cognitivas y emocionales expresadas por un trabajador en el desarrollo de sus obligaciones (Kahn, 2018). El sujeto engaged es enérgico, eficaz en la labor encomendada, con elevada energía, entusiasta, comparte sus conocimientos actuando de forma comprometida, con plenitud y autorrealización (... ). Además de las cualidades indicadas presenta diversas categorías: energíavitalidad, implicación-compromiso y eficacia en el trabajo (Ruiz, 2017). Implica sentimientos de plenitud, dedicación y entrega por las ocupaciones, aún en medio de la adversidad se mantiene el empeño y la tenacidad por las ocupaciones (Maury, Martínez y González, 2016).

Se caracteriza por el Vigor, la Dedicación y la Absorción en el trabajo. El vigor se vincula a altos niveles de energía, resiliencia y alto esfuerzo en las actividades incluso cuando aparecen obstáculos en el camino. La dedicación supone una alta implicación laboral, sentimientos de significación, entusiasmo, inspiración, orgullo y reto por el trabajo realizado. La absorción es el estado mental que ocurre cuando se está totalmente concentrado en el trabajo, se experimenta que el tiempo 'pasa volando', acompañado de fuertes dosis de disfrute y placer (Pena y Extremera, 2012).

El engagement o compromiso tiene sus bases en la psicología positiva, y ha sido estudiado en el contexto académico, donde se le ha definido como una condición afectiva, positivista (García, Labajos y Fernández, 2015) y motivacional vinculada al área estudiantil (Macarena, Aja y Plaza, 2018; Montoya y Moreno, 2012). Los estudiantes mantienen un estado psicológico de bienestar, dedicación y esfuerzo ante los estudios (Caballero, Hederich y García, 2015), muestran un alto desempeño, siendo perseverantes, participativos y comprometidos con sus aspiraciones académicas (Medrano, Moretti y Ortiz, 2015), con capacidad de adaptarse a sus docentes, compañeros y ambiente en general.

A nivel universitario, suele demostrarse en alumnos intelectuales, participativos, que mantienen objetivos claros en su vida y que se encuentran involucrados activamente en su carrera (McInnis et al., 2000). De esta manera, el engagement académico combina el desempeño y el nivel favorable de integración en la universidad (Horstmanshof y Zimitat, 2007); los alumnos muestran rasgos de autoeficacia, satisfacción académica y permanencia en los estudios (Schaufeli et al., 2002; Bresó, Llorens, y Salanova, 2005, Shih, 2012); así como interés y disfrute por su aprendizaje lo que impacta favorablemente en los resultados de las evaluaciones, beneficiando 
el aprendizaje en esta etapa (Mih y Mih, 2013; Martin, 2008).

Portalanza, Grueso y Duque (2017) señalan que el engagement presenta un componente conductual y psicológico. El primero orienta al estudiante a buscar consejos en su docente, emplea horas fuera de clases para sus actividades académicas y desarrolla un aprendizaje significativo. Estas conductas evidencian responsabilidades que superan el ámbito del aula universitaria (Horstmanshof y Zimitat, 2007; Hughes, et al., 2009). El componente psicológico se centra en respuestas afectivas orientadas al aprendizaje. Se refleja en sentimientos de autovalía por los logros alcanzados y también negativas producto del cansancio que experimenta el estudiante (Medrano, Moretti y Ortiz, 2015). Tiene también una naturaleza interindividual, ya que este estado se integra y comparte con los miembros del grupo, dando lugar al engagement colectivo (Salanova y Schaufeli, 2009).

\section{Desempeño académico}

La universidad está llamada a formar y transformar, permitiendo a los estudiantes la adquisición de competencias que desarrollen un saber analítico y crítico (López, 2016). El desempeño académico hace referencia al rendimiento adquirido en el ámbito escolar o universitario. Representa una medida de las capacidades del alumno, que expresa lo que éste ha aprendido a lo largo del proceso formativo (Willcox, 2011). También puede definirse como el nivel de conocimientos demostrado en un área comparado con la norma de edad y nivel académico (Jiménez, 2000), siendo el producto entre lo obtenido en el centro de enseñanza y el esfuerzo empleado para obtenerlo, expresado en una calificación (Lamas, 2015). Es un fenómeno vigente, porque determina la calidad y la cantidad de los aprendizajes (Ruiz, 2002), y multidimensional, ya que integra diversos componentes: cognitivo-afectivo-conductualinstitucional (Fernández, 2010), donde se ponen en evidencia las aptitudes vinculadas a aspectos volitivos, afectivos y emocionales para responder a los objetivos institucionales (Reyes, 2003; Díaz, 1995).

El desempeño académico incluye varios componentes: los procesos de aprendizaje, aspectos cognitivos, estructura educativa, mostrando variaciones de acuerdo a las condiciones orgánicas $\mathrm{y}$ ambientales que determinan las aptitudes y experiencias. Se ve influenciado por el nivel intelectual, personalidad, motivación, hábitos de estudio, intereses y autoestima (Martí, 2003). Es un producto condicionado por múltiples variables (Nieto, 2008). López (2009), citado por Ramón (2013) indica como factores que influyen en el desempeño los aspectos intelectuales (capacidades, aptitudes e inteligencia); aspectos psíquicos, como la personalidad, la motivación, el auto concepto, la adaptación, ya que los fracasos escolares se dan con mayor frecuencia en alumnos que viven con problemas emocionales y afectivos; aspecto socio ambiental: representado por la influencia que ejercen los condicionantes ambientales que rodean al alumno (familia, el barrio y estrato social) y aspecto pedagógico que incluye los problemas de aprendizaje.

Varios autores sostienen la necesidad de emplear múltiples estrategias de evaluación para determinar el nivel de desempeño de los estudiantes (Schwartz, 1992; Schuwartz, 1994; Hull, 1995; Collins y Gamble, 1996; Schuwirth y Van der Vleuten, 2004; Carr, 2004; Van der Vleuten y Schuwirth, 2005). De igual manera, los planes y programas de estudio deben especificar con claridad qué aprendizaje se debe lograr y cómo evaluarlo para que la evaluación sea útil (Wass, 2001). No obstante, lo verdaderamente importante es definir los objetivos educativos a que aspiramos, analizar los contextos y las dificultades para generar propuestas y mecanismos de acción que permitan ir avanzando en este camino.

\section{METODOLOGÍA}

La investigación se realizó en el ámbito de la Facultad de Ciencias Aplicadas de la Universidad Nacional del Centro del Perú con estudiantes de las carreras profesionales de administración de negocios, administración hotelera y turismo e ingeniería agroindustrial, en Pomachaca Tarma, Junín, Perú-2018.

El estudio fue de nivel correlacional, diseño no experimental, transeccional, el cual se operativizó a través de la escala validada internacionalmente UWES de engagement académico (Medrano, Moretti y Ortiz, 2015), los ítems de cada factor presentan una elevada homogeneidad observándose así una óptima consistencia interna (valores $\alpha$ comprendidos entre ,80 y ,90), con una alta relación (superiores a $r=, 65$ ) 
entre sus componentes (Salanova et al., 2000). El cuestionario evaluó la variable engagement académico en las dimensiones: vigor, dedicación y absorción; para la evaluación del desempeño académico se consideraron los promedios consolidados de tres carreras profesionales. Estuvo dirigido a la totalidad de estudiantes, compuestos por una población-muestra de 298 alumnos de las tres carreras profesionales de la Facultad de Ciencias Aplicadas, considerados de acuerdo a las fichas de matrículas.

Con el objeto de comprobar la asociación de variables se empleó el coeficiente de correlación de Spearman para muestras no paramétricas cualitativas categóricas, el nivel de significancia fue de $5 \%$, y el de confianza fue de $95 \%$. Se utilizó la prueba estadística Neamer, no paramétrica y para el análisis inferencial de los resultados ChiCuadrada. Los datos fueron procesados en el paquete estadístico SPSS 22.

Los estudiantes fueron convocados e informados por los investigadores obteniéndose los permisos pertinentes. El proceso tuvo en consideración consideraciones éticas nacionales e internacionales. Fue aprobado por el comité de ética de la universidad.

\section{RESULTADOS Y DISCUSIÓN}

El hallazgo más importante, es que no existe correlación significativa entre engagement $y$ el desempeño académico, a partir del cual se contrasta con la evidencia empírica, existiendo un nivel apropiado de motivación y responsabilidad en los estudiantes de la Facultad respecto a sus estudios.

Se buscó demostrar la existencia de correlación significativa entre habilidades sociales de engagement y desempeño académico en estudiantes de la Facultad de Ciencias Aplicadas, se obtuvo un p-valor de 0.33 , por tanto, se infiere que no existe correlación significativa entre engagement y desempeño académico, así mismo el coeficiente de correlación 0.123 , indica un nivel bajo de asociación entre estas variables.

En la Escuela Profesional de Ingeniería Agroindustrial, el mejor desempeño académico lo tuvieron los estudiantes del IX semestre con 13.19 en promedio, seguido del VII semestre con 12.36, V semestre con 11.90 y el III semestre con 10.40; así mismo todos los estudiantes (82) tuvieron un promedio de 12.51. En Administración Hotelera y Turismo, el mejor desempeño académico estuvo en el V semestre con 14.46 en promedio, seguido del IX semestre con 14.06, VII semestre con 13.39 y el III semestre con 13.32; asimismo todos los estudiantes (78) tienen un promedio de 13.87. En Administración de Negocios, el mejor desempeño estuvo en el IX semestre con 14.67 en promedio, seguido del V semestre con 13.68, III semestre con 13.58 y el II semestre con 13.32, asimismo todos los estudiantes (138) tienen un promedio de 13.79 .

Tabla 1.

Desempeño académico de estudiantes de la Facultad de Ciencias Aplicadas.

\begin{tabular}{cccccc}
\hline & N & Mínimo Máximo & Media & $\begin{array}{c}\text { Desviación } \\
\text { estándar }\end{array}$ \\
\hline $\begin{array}{c}\text { Promedio de } \\
\text { Notas }\end{array}$ & 298 & 2.30 & 17.41 & 13.4584 & 1.94231 \\
$\begin{array}{c}\text { N válido (por } \\
\text { lista) }\end{array}$ & 298 & & & & \\
\hline
\end{tabular}

De los 298 estudiantes de la Facultad de Ciencias Aplicadas, de las 3 carreras profesionales en estudio la nota mínima es de 2.30 y la máxima de 17.41, con una media de 13.46 y desviación estándar de 1,94 (Tabla 1).

Rendir académicamente implica alcanzar un determinado nivel de conocimiento, el cual es medido en una evaluación que considera como criterio una calificación (Solano, 2015). Los hallazgos de Mizola (2005), muestran que el esfuerzo puesto por el estudiante universitario, sus conocimientos previos y competencias son factores que afectan el desempeño académico, considerando también la presencia de factores institucionales internos propios de las universidades (Agudelo, Martínez \& Ortiz, 2013).

Cuando el desempeño se ubica por debajo de lo esperado genera insatisfacción en los estudiantes, pudiendo estos resultados estar vinculados a metodologías didácticas (Chipana, 2012; Lamas, 2015). Por otro lado, los estudiantes con alto desempeño académico desarrollan mayores probabilidades para permanecer en la universidad (Houn y Sankey, 2000; Potts, Schultz y Foust, 2003; Krause, et al., 2005). Abandonar los estudios genera efectos no sólo en el desarrollo de los aprendizajes, sino en algunos componentes de las habilidades sociales como la motivación y confianza personal impactando en el escaso logro de mejores aprendizajes (Reyes, 2007). 
Tabla 2.

Habilidades sociales en Engagement en estudiantes de la Facultad de Ciencias Aplicadas.

\begin{tabular}{llll}
\hline & Nivel & Fi & \% \\
\hline \multirow{2}{*}{ Habilidades sociales en engagement } & Bajo & 141 & 47.3 \\
& Alto & 157 & 52.7 \\
& Total & 298 & 100 \\
\hline
\end{tabular}

El $52.7 \%$ de los estudiantes universitarios (157) tienen un nivel alto de Habilidades Sociales en engagement académico, demostrando vigor, dedicación y absorción, con lo cual se evidencia una alto esfuerzo y persistencia, acompañado de un sentido y entusiasmo por las actividades que realizan. Resultados similares reflejan recientes investigaciones (Dávila, Retamozo y Vásquez, 2015; Zúñiga, 2017; Arroyo, 2018) poniendo en evidencia que éstas influyen en la forma de afrontar las situaciones que se presentan en la vida personal y académica, siendo un pilar fundamental en cualquier escenario.

Tabla 3.

Prueba de Kolmogorov-Smirnov de Habilidades sociales en Engagement y desempeño académico

\begin{tabular}{|c|c|c|c|c|}
\hline & \multicolumn{2}{|l|}{$\begin{array}{l}\text { Kolmogorov- } \\
\text { Smirnov }^{\mathrm{a}}\end{array}$} & \multicolumn{2}{|l|}{ Shapiro-Wilk } \\
\hline & \begin{tabular}{l|l|} 
Estadístico & $\mathrm{g}$ \\
\end{tabular} & Sig. & \begin{tabular}{|l|l|} 
Estadísticoco & $\mathrm{gl}$ \\
\end{tabular} & Sig. \\
\hline $\begin{array}{l}\text { Hab. Sociales en Engagement } \\
\text { y desempeño académico }\end{array}$ & $.289 \quad 2$ & $298 \mathbf{0 . 0 0 0}$ & .808 & 0.000 \\
\hline Prom.Notas.Facap.Tablas & .368 & 2980.000 & .705 & 0.000 \\
\hline
\end{tabular}

Los datos permiten afirmar que estamos ante una muestra no paramétrica propia de las variables categóricas.

Tabla 4.

Correlación de Spearman Vigor y desempeño Académico

\begin{tabular}{|lll|}
\hline Dimensión de variable & $\begin{array}{l}\text { p- } \\
\text { valor }\end{array}$ & $\begin{array}{l}\text { Coeficiente de } \\
\text { correlación }\end{array}$ \\
\hline $\begin{array}{l}\text { Dimensión Vigor de } \\
\text { Engagement y Rendimiento } \\
\text { Académico }\end{array}$ & 0.29 & .127 \\
\hline
\end{tabular}

En la dimensión vigor de engagement los estudiantes muestran altos niveles de energía y resiliencia, voluntad de dedicar esfuerzos, no fatigarse con facilidad y persistencia ante las dificultades. El vigor y desempeño académico no se correlacionan significativamente al haber obtenido un p-valor de 0.29 y un coeficiente de correlación de Spearman de .127, (Tabla 4) contradiciendo lo establecido por Parra (2011) quien refiere que la satisfacción se conceptualiza como una dimensión del engagement, favoreciendo al rendimiento del estudiante y un mejor afrontamiento de situaciones estresantes.

Tabla 5.

Correlación de Spearman Dedicación y desempeño académico

\begin{tabular}{lll}
\hline Dimensión de variable & p-valor & $\begin{array}{l}\text { Coeficiente de } \\
\text { correlación }\end{array}$ \\
\hline $\begin{array}{l}\text { Dimensión Dedicación de } \\
\text { engagement y Rendimiento }\end{array}$ & 0.115 & .091 \\
Académico & & \\
\hline
\end{tabular}

Sobre la dimensión dedicación de engagement, los alumnos encuentran sentido a las actividades que realizan, acompañados de entusiasmo y orgullo. La dedicación y desempeño académico tampoco encuentran correlación significativa con un p-valor de 0.115 y un coeficiente de correlación de Spearman de 0.91, (tabla 5) refutando lo expresado por Gonzales, Caso, Díaz, y López (2012) quienes precisan que el desempeño académico tiene que ver principalmente con reto, logro y resultados académicos.

Tabla 6.

Correlación de Spearman Absorción y desempeño académico

\begin{tabular}{lll}
\hline Variable/Dimensión & p-valor & $\begin{array}{l}\text { Coeficiente de } \\
\text { correlación }\end{array}$ \\
\hline $\begin{array}{l}\text { Dimensión Absorción de } \\
\text { Engagement y Rendimiento } \\
\text { Académico }\end{array}$ & 0.38 & .120 \\
\hline
\end{tabular}

En la dimensión absorción de engagement y desempeño académico, se obtuvo un p-valor de 0.38 y un coeficiente de correlación de Spearman .120 (Tabla 6), no existiendo correlación significativa, discrepando con Cominetti y Ruiz (1997) citado por Edel (2003) quienes refieren que las expectativas del entorno directo del estudiante permiten sacar a relucir los paradigmas positivos $\mathrm{y}$ negativos que acompañan al estudiante y que serán determinantes en su rendimiento y logros académicos.

Tabla 7.

Correlación de Spearman Habilidades sociales en engagement y desempeño académico

\begin{tabular}{lll}
\hline Variables & p-valor & $\begin{array}{l}\text { Coeficiente de } \\
\text { correlación }\end{array}$ \\
\hline $\begin{array}{l}\text { Habilidades sociales en } \\
\text { Engagement y desempeño } \\
\text { académico. }\end{array}$ & 0.33 & $0.123 *$ \\
* La correlación es significativa en el nivel 0,05 (bilateral). Con un p-valor del 0.33 \\
$\begin{array}{l}\text { y coeficiente de correlación } 0.123, \text { se concluye que no existe correlación } \\
\text { significativa entre las habilidades sociales de engagement y desempeño académico } \\
\text { en estudiantes de la Facultad de Ciencias Aplicadas (Tabla 7). }\end{array}$
\end{tabular}


Respecto al propósito principal del estudio se afirma que las habilidades sociales en engagement no tienen relación significativa con el desempeño académico, lo cual evidencia que los estudiantes presentan niveles adecuados de vigor, dedicación y absorción, mostrando interés por aprender, estudiar e investigar, reafirmando lo expresado por Fenollar, Román y Cuestas (2007) quienes concluyen que no existe relación significativa entre resultados esperados y calificaciones promedio.

Al mismo tiempo, los resultados refutan lo referido por Harackiewicz, et al. (2000), quienes hallaron que la combinación de ambas variables puede tener consecuencias beneficiosas dentro del contexto educativo, lo cual no es concluyente en este estudio, pero sí se aprecia que el engagement académico es un elemento neurálgico para promover aspectos psicológicos positivos en el aprendizaje de los estudiantes, coincidiendo con Martin (2008) y Salanova y Schaufeli (2009).

Los estudios desarrollados sobre habilidades sociales y desempeño académico han revelado resultados poco consistentes que permitan reflejar que exista una relación causal entre estas variables (Lewis y Taylor, 2007). Tal es el caso de nuestros hallazgos donde se evidencia que no existe tal relación, al igual que en el estudio de Ortiz, et al. (2018) donde se determinó una correlación negativa inversa sin asociación estadísticamente significativa $(r=0,073, p<0,135)$.

En otras investigaciones se concluye que altos niveles de aprendizaje se vinculan a habilidades sociales avanzadas (Kholin et al., 2016; García, 2015). También existe asociación fuerte y directa entre la autoestima y el rendimiento académico de los estudiantes universitarios (Acuña, 2013; Nicho, 2013); no obstante, otras investigaciones señalan lo opuesto (Chilca, 2017). Se comparten estos criterios, ya que los hallazgos muestran adecuadas habilidades sociales en engagement de los estudiantes; sin embargo, si éstas aumentan no necesariamente se incrementa su rendimiento académico en igual proporción, respondiendo a fenómenos causales. Este criterio se pone de manifiesto en múltiples investigaciones (Cañabate et al., 2018; Rytkönen et al., 2012). Adicionalmente, aunque algunas investigaciones sugieren que el engagement se puede ver facilitado por la relación con los docentes y el clima escolar (Martin, 2008; Mih y Mih, 2013, Shih, 2012), no son muchos los estudios realizados en este contexto que avalen estas afirmaciones.
Es oportuno señalar el planteamiento de Mizola (2005) sobre la necesidad de medir el desempeño académico y definir las estrategias para su mejoramiento revisando los elementos que influyen en éste, considerando que el desempeño es una variable que depende de diversos factores. A partir de lo anterior, Edel (2003) señala cuatro aspectos que intervienen en el desempeño académico: los insumos, la motivación escolar, el autocontrol del alumno y las habilidades sociales. Se considera que estos aspectos requieren ser valorados en los contextos universitarios para determinar sus incidencias en el desempeño académico.

Las habilidades sociales en engagement son necesarias para que el estudiante universitario se desarrolle en este contexto, pero no son indispensables para obtener un adecuado desempeño académico. Las mediciones realizadas en este estudio no controlaron estrictamente los factores que pudieron estar presentes en la correlación estudiada; por lo que se deben efectuar investigaciones que tienen que pasar a un siguiente nivel para poder explicar el origen de la relación débil de las variables estudiadas y sus posibles causas.

Con este trabajo se abren nuevos horizontes para el estudio del engagement académico junto a otras variables vinculadas al contexto universitario y a procesos personales de los estudiantes. Ello permitirá profundizar en el conocimiento relativo a estos temas.

\section{CONCLUSIONES}

La investigación ha demostrado que no existe una relación significativa entre las habilidades sociales de engagement y el rendimiento académico, con lo cual podría preverse que en el futuro y de manera súbita e inesperada los estudiantes pierdan el interés en sus estudios y en consecuencia bajen sus calificaciones cíclicamente o abandonen sus estudios.

Los estudiantes muestran adecuadas habilidades sociales de engagement en la dimensión vigor, desempeñándose con altos niveles de energía y resistencia mental; no obstante, estas conductas no se relacionan con el desempeño académico. El engagement en las dimensiones dedicación y absorción no se relacionan con el desempeño académico. El entusiasmo por los desafíos académicos, así como la concentración y 
satisfacción por estas actividades no determinan los logros de aprendizaje.

El logro de un comportamiento académico óptimo requiere interés y compromiso activo del estudiante, pero pueden surgir situaciones adversas que no necesariamente afectan el rendimiento. Adicionalmente, la presencia de múltiples factores protectores puede contribuir con un adecuado desempeño y bienestar psicológico. Aun cuando los resultados académicos no sean los esperados; es importante valorar y sentirse comprometido emocionalmente con los estudios, demostrando vigor, energía, dedicación, entusiasmo y concentración; ello permitirá una mejor disposición al aprendizaje, previniendo conductas desadaptativas que incrementen la vulnerabilidad del estudiante.

\section{Agradecimiento}

A la comunidad universitaria de la facultad de Ciencias Aplicadas de la Universidad Nacional del Centro, sede Tarma, Junín. A la Escuela de Post grado de la Universidad Nacional Hermilio Valdizán por su apoyo incondicional en el desarrollo de la investigación.

\section{Conflicto de intereses}

Los autores declaran no tener conflictos de interés, en relación a la información, respecto a engagement y desempeño académico.

\section{REFERENCIAS BIBLIOGRÁFICAS}

Acuña, J. (2013). Autoestima y rendimiento académico de los estudiantes del X ciclo 2012 - II de la escuela académica profesional de educación primaria y problemas de aprendizaje de la Universidad Nacional José Faustino Sánchez Carrión - Huacho (Tesis de Maestría). Universidad Nacional José Faustino Sánchez Carrión, Lima.

Agudelo, C., Martínez, L., \& Ortiz, I. (2013). Gestión del conocimiento: un activo intangible a través de la investigación. Praxis, 9, 108113. En: http://revistas.unimagdalena.edu.co/ index.php/praxis/article/view/750/694.

Betancourth, S., Zambrano, C., Ceballos, A., Benavides, V., \& Villota, N. (2017). Habilidades sociales relacionadas con el proceso de comunicación en una muestra de adolescentes. Revista Virtual de Ciencias
Sociales y Humanas "PSICOESPACIOS", 133-148.

Borges del Rosal, Á., Hernández-Jorge, C. M., \& González Sierra, M. A. (2012). Achievement predictors in a secondary students' sample. Quality \& Quantity, 46(6), 1687-1697. https://doi.org/10.1007/s11135-011-9547-5

Bresó, E., Llorens S., \& Salanova, M., (2005). Creencias de eficacia académica y engagement en estudiantes universitarios. Jornadas de Fomento de la Investigación. www.uji.es/bin/ publ/edicions/jfi9/psi/6

Brown Kirschman, J. K., Johnson, R. J., \& Roberts, M. C. (2008). Positive psychology for children and adolescent: Development, prevention, and promotion, Handbook of positive psychology (2nd ed., pp. 133-148). New York: Oxford.

Caballero, C., Hederich, C. y García, A. (2015). Relación entre Burnout y Engagement académicos con variables sociodemográficas y académicas. Psicología desde el Caribe; 32(2): 254-267. En: http://www.scielo.org.co/ $\mathrm{pdf} / \mathrm{psdc} / \mathrm{v} 32 \mathrm{n} 2 / \mathrm{v} 32 \mathrm{n} 2 \mathrm{a} 05 . \mathrm{pdf}$

Caballo, V., Salazar, I., Irurtia, M. y Olivares, P. (2014). Relación de las habilidades sociales con la ansiedad social y los estilos/trastornos de la personalidad. Psicología Conductual, 22, 401-422.

Cañabate, D., Martínez, G., Rodríguez, D. \& Colomer, J. (2018). Analysing Emotions and Social Skills in Physical Education. Sustainability, 10(5), 1-8. https://doi. org/10.3390/su10051585

Cacioppo, J., Cacioppo, S., \& Cole, S. (2013). Neurociencia Social y Genómica Social: El Surgimiento del Análisis Interdisciplinario Multinivel. International Journal of Psychological research, 1-6.

Carr, S. J. (2004). “Assessing clinical competency in medical senior house officers: how and why should we do it?", en Postgrad Med J, 80.

Chávez, M., Contreras, O. y Velázquez, M. (2013). Adaptación y pensamiento constructivo en estudiantes universitarios. Psicogente, 16 (30): pp. 311-323. Recuperado de https://goo. g1/LRrzWJ.

Chilca, M.L. (2017). Autoestima, hábitos de estudio y rendimiento académico en estudiantes universitarios. Propósitos y Representaciones, [S.1.], v. 5, n. 1, p. 71-127, abr. 2017. ISSN 2310-4635. En: https://revistas.usil.edu.pe/ index.php/pyr/article/view/145/377

doi: http://dx.doi.org/10.20511/pyr2017.v5n1.145. 
Chipana, F.S. (2012). Rendimiento académico. Revista de Investigación Scientia, 2(1), 83101.

Compagnucci, E., Cardós, P., Denegri, A., \& Barboza, C. (2002). El adolescente frente al conocimiento. Orientación y Sociedad, (3), 93-104. Retrieved from http://www.scielo. org.ar/pdf/orisoc/v3/v3a06.pdf.

Collins, J. P. \& Gamble, G. D. (1996). "A multiformat interdisciplinary final examination", en Med Educ, 30.

Del Rosal, B., González, M., Aceituno, A., Martín, J.C. y Arjona, A. (2012). Efecto de la implantación de turnos de enfermería "a demanda" sobre las horas de absentismo. Gaceta Sanitaria, 26, pp. 480-482.

De Onzoño, S.I. (2016). Talent Management and Sustainable Companies. Cosmopolitan Managers: Executive Development that Works (pp. 203-221). https://doi.org/10.1057/978-1137-54909-9 12.

Edel, R. (2003). El rendimiento académico: concepto, investigación y desarrollo. REICE Revista Interamericana sobre Calidad, Eficacia y Cambio en Educación, 1(2), 1-15. En http://www.ice.deusto.es/RINACE/reice/ volln2/Edel.pdf

Fenollar, P., Cuestas, P.J. \& Román, S. (2007). University students' academic performance: An integrative conceptual framework and empirical analysis. British Journal of Educational Psychology, 77, 873-891.

Fernández, M. (2010). Variables predictoras del rendimiento académico en estudiantes de estadística. Facultad de Ciencias de la Educación de la Universidad de Carabobo. Documento de investigación. Venezuela.

García, C. (2005). Habilidades sociales, clima social familiar y rendimiento académico en estudiantes Universitarios. Revista de Psicología Liberabit. 11, 63-74.

García, J., Labajos, T. y Fernández, F. (2015). Los estudiantes de Grado en Enfermería y su compromiso con los estudios. Revista electrónica trimestral de Enfermería; 14(38):169-177. En: http://scielo.isciii.es/ pdf/eg/v14n38/docencia4.pdf

Gismero, E. (2010). Manual de la Escala de Habilidades Sociales ( $3^{\mathrm{a}}$ ed.). Madrid: TEA, Ediciones, S.A.

Gonzales, C., Caso, J., Díaz, K. y López, M. (2012). Rendimiento académico y factores asociados. Aportaciones de algunas evaluaciones a gran escala. Revista Bordón 64 (2), 2012, 51-68,
ISSN: 0210-5934.

Guadalupe, N. (2015). Clima social familiar $y$ rendimiento académico en estudiantes del cuarto grado del nivel secundario. Institución educativa San Juan de la Libertad. Chachapoyas. (Tesis de Grado). Universidad Nacional Toribio Rodríguez De Mendoza, Amazonas.

Guàrdia, J., Freixa, M., Peró, M., Turbany, J., Cosculluela, A., Barrios, M., \& Rifà, X. (2006). Factors Related to the Academic Performance of Students in the Statistics Course in Psychology. Quality \& Quantity, 40(4), 661-674. https://doi.org/10.1007/ s11135-005-2072-7

Harackiewicz, J.M., Barron, K.E., Tauer, J.M., Carter, S.M. y Elliot, A.J. (2000). Short-term and long-term consequences of achievement goals: predicting interest and performance over time. Journal of Educational Psychology, 92, 316-330.

Herrera, A., Freytes, M., López, G. y Olaz, F. (2012). Un estudio comparativo sobre las habilidades sociales en estudiantes de Psicología. International Journal of Psychology and Psychological Therapy, 12(2), 277-287.

Horstmanshof, L. y Zimitat, C. (2007). Orientación del tiempo futuro predice compromiso académico entre los estudiantes universitarios de primer año. Revista británica de psicología educativa; 77(3): 703-18. En: https://www. ncbi.nlm.nih.gov/pubmed/17908382

Hull, A. L. (1995). "Validity of three clinical performance assessments of internal medicine clerks", Acad Med, 70.

Hughes, J., Wen, L., Kwok, O. y Loyd, L. (2009) Apoyo profesor-alumno, compromiso comprometido y Logro: un estudio longitudinal de 3 años. Instituto Nacional de Salud. Revista; 100(1): 1-14. En: https://www. ncbi.nlm.nih.gov/pmc/articles/PMC2705122/ pdf/nihms83519.pdf

Huon, G. y Sankey, M. (2000). The transition to University: Understanding differences in success. Papers presented at the 4th Pacific Rim year in higher education conference, Queensland, Australia.

Jiménez, M. (2000). Competencia social: intervención preventiva en la escuela. España: Infancia y Sociedad: Revista de estudios, 1(3), 122-137

Kahn, W. (2018). Condiciones psicológicas de compromiso personal y desconexión en el trabajo. Revista de Administración; 30(3): 
201-205. En: https://journals.aom.org/doi/ pdf $/ 10.5465 / 256287$

Kholin, M., Meurs, J. A., Blickle, G., Whiler, A., Ewen, C., \& Momm, T. D. (2016). Refining the Openness-Performance Relationship: Construct Specificity, Contextualization, Social Skill, and the Combination of Trait Selfand Other-Ratings. Journal of Personality Assessment, 98(3), 277-88. https://doi.org/10. 1080/00223891.2015.1076430

Krause, K., Hartley, R., James, R., y McInnis, C. (2005). The first year experience in Australian Universities: Findings from a decade of National studies. Melbourne: Centre for the Study of Higher Education, University of Melbourne.

Lamas, H. (2015). Sobre el rendimiento escolar. Propósitos y Representaciones, 3(1), 313386. doi: 10.20511/pyr2015.v3n1.74

León, B., Felipe, Polo y Palacios (2016). Elementos mediadores en la eficacia del aprendizaje cooperativo: entrenamiento previo en habilidades sociales y dinámica de grupos. Anales de psicología, 22, 105-112.

León, B., Castaño, Mendo \& Iglesias. (2015). Habilidades sociales en equipos de aprendizaje cooperativo en el contexto universitario. Psicología Conductual, 23(2), 191-214.

Lewis, T. y Taylor, P. (2007). Social Inequality in Education: A Constraint on an American High-Skills. Curriculum Inquiry, 37(4), 329-349. https://doi.org/10.1111/j.1467873X.2007.00390.

86 López, M. A. (2016). La gestión educativa en el marco de la pedagogía crítica. Módulo Maestría Educación Docencia Universidad de Manizales.

Macarena, M., Aja, J. y Plaza, J. (2018). Estrés percibido en estudiantes: influencia del burnout y del engagement académico. Revista Internacional de Investigación e Innovación Educativa; 9(1): 220-236. En: https://dialnet. unirioja.es/servlet/articulo?codigo $=6182971$

Martí, E. (2003). Representar el mundo externamente. La construcción infantil de los sistemas externos de representación. Madrid: Antonio Machado.

Martin, A.J. (2008). Enhancing student motivation and engagement: The effects of a multidimensional intervention. Contemporary Educational Psychology, 33, 239-269.

Maury, J., Martínez, M. y González, Z. (2016). Relación del optimismo, la personalidad resistente y el engagement con el trabajo en una muestra de empleados. Revista Puertorriqueña de Psicología; 25(2): 280296. En: https://dialnet.unirioja.es/descarga/ articulo/4895943.pdf\%0A\%0A

McCarthy, B. y Trace, A. (2014). O'Donovan Integrating psychology with interpersonal communication skills in undergraduate nursing education: Addressing the challenges. Nurse Educ Pract., 14 (2014), pp. 227-232

McInnis, C., James, R., \& Hartley, R. (2000). Trends in the first year experience. Canberra: DETYA. Higher Education Division.

Medrano, L., Moretti, L. y Ortiz, A. (2015). Medición del engagement académico en estudiantes universitarios. Revista Iberoamericana de Diagnóstico y Evaluación Psicológica; 2(40): 114-124. En: http://www. redalyc.org/pdf/4596/459645432012.pdf

Mendo, L., León, B., Felipe, E. y Polo, M. (2016). Entrenamiento en habilidades sociales en el contexto universitario: efectos sobre las habilidades sociales para trabajar en equipo y la ansiedad social. Psicología Conductual, 24(3), 423-438.

Mih, V., \& Mih, C. (2013). Perceived autonomysupportive teaching, academic selfperceptions and engagement in learning: Toward a process model of academic achievement. Cognition, Brain \& Behavior. An Interdisciplinary Journal, 4, 289-313.

Mizola, A. (2005). Factores que Inciden en el rendimiento escolar en Bolivia. (Tesis de pregrado) Universidad de Chile.

Montoya, P., y Moreno, S. (2012). Relación entre síndrome de burnout, estrategias de afrontamiento y engagement. Psicología desde el Caribe; 29(1): 205-227. En: http:// www.redalyc.org/pdf/213/21323171011.pdf.

Morales, M., Benitez, M., \& Agustín, D. (2013). Habilidades para la vida (cognitivas y sociales) en adolescentes de una zona rural. Revista Electrónica de Investigación Educativa, 98113.

Navarro, R. (2003). Clima social familiar y habilidades sociales en estudiantes del cuarto año de educación secundaria de la Institución Educativa Parroquial Santa Catalina de la ciudad de Juliaca. (Tesis para optar el título profesional de Psicólogo) Universidad Peruana Unión, Juliaca - Perú.

Nicho, A. (2013). Relación entre la autoestima y el rendimiento académico de los estudiantes de la E.B.C. tecnológica de la facultad de 
educación de la Universidad Nacional José Faustino Sánchez Carrión (Tesis inédita). Universidad Nacional José Faustino Sánchez Carrión, Lima.

Nieto, S. (2008). Hacia una teoría sobre el rendimiento académico en enseñanza primaria a partir de la investigación empírica: datos preliminares. Teoría de la Educación, 20, 249-274.

Ng, C., Bartlett, B., \& Elliott, S.N. (2018). "Opportunity to Connect": Social Skills as Engagement Agents. En C. Ng, B. Bartlett, \& S. N. Elliott (Eds.), Empowering Engagement: Creating Learning Opportunities for Students from Challenging Backgrounds (pp. 67-89). https://doi.org/10.1007/978-3-319-9465284

Ortiz, W., Santos-Morocho, J., Baculima, V. y Cabrera, J. (2018). Habilidades sociales y rendimiento académico en estudiantes de Psicología. Revista Cientifica Digital de Psicología PSIQUEMAG Vol. 7, $\mathrm{N}^{\circ}$ 1, 2018. En: https://www.researchgate. net/publication/333114783_Habilidades sociales_y rendimiento_academico en estudiantes de Psicologia.

Pades, A. y Roso, F. (2013). Tutoría académica y evaluación formativa de programa de Entrenamiento Habilidades Sociales (EHS) en Grado de Enfermería Docente, 100, pp. 4-10. View Record in ScopusGoogle Scholar

Parada, M., \& Pérez, C. E. (2014). Relación del engagement académico con características académicas y socioafectivas en estudiantes de Odontología. Educación Médica Superior, 28(2), 199-215. Retrieved from http://scielo.sld.cu/scielo.php?script=sci arttext\&pid=S0864-21412014000200003\&1n $\mathrm{g}=\mathrm{es} \& n \mathrm{~mm}=\mathrm{iso} \& \mathrm{tlng}=\mathrm{es}$

Parra, P. (2011). Relación entre el nivel de Engagement y el rendimiento teórico/ práctico. (Tesis de maestría). Concepción, Chile: Universidad de Concepción.

Párraga, J.M. (2005). Eficacia del programa I.R.I.S. para reducir el sindrome de burnout y mejorar las disfunciones emocionales en profesionales sanitarios [tesis doctoral]. Universidad de Extremadura.

Patrício, M., Maia, F., \& Bezerra, C. (2015). Las habilidades sociales y el comportamiento infractor en la adolescencia. Subjetividad y Procesos Cognitivos, 17-38.

Pena, M., \& Extremera, N. (2012). Inteligencia emocional percibida en el profesorado de
Primaria y su relación con los niveles de burnout e ilusión por el trabajo (engagement). Revista de Educación (359), Sep-Dic. 604627

Portalanza, C., Grueso, M. y Duque, E. (2017). Propiedades de la Utrecht Work Engagement Scale (UWES-S 9): Análisis exploratorio con estudiantes en Ecuador. Innovar. Revista de Ciencias Administrativas y Sociales; 27(64): 145-155. En: https://revistas.unal.edu.co/ index.php/innovar/article/view/62374/60525.

Potts, G., Schultz, B., y Foust. (2003). The effect of freshman cohort groups on academic performance and retention. Journal of College Student Retention, vol.5, n.4, 385-395.

Ramón, J. (2013). El desempeño docente y su incidencia en el rendimiento académico de los estudiantes de segundo a sexto grados de educación general básica. (Tesis de pregrado) Ambato: Universidad Técnica de Ambato. En: https://repositorio.uta.edu.ec/ jspui/handle/123456789/6920

Riquelme, E., y Munita, F. (2013). Lectura mediada y escenarios paradigmáticos ficticios. "Actas del Seminario Internacional ¿Qué leer? ¿Cómo leer?, Perspectivas sobre la lectura en la Infancia”. (pp.349-364). Chile: Ministerio de Educación de Chile.

Rytkönen, H., Parpala, A., Lindblom-Ylänne, S., Virtanen, V. \& Postareff, L. (2012). Factors affecting bioscience students' academic achievement. Instructional Science, Vol.40 (2), 241- 256. https://doi.org/10.1007/s11251011-9176-3

Rodríguez, L.Y., Cacheiro, M.L. y Gil, J.A. (2014). Desarrollo de habilidades sociales en estudiantes mexicanos de la preparatoria a través de actividades virtuales en la plataforma Moodle. Education in the Knowledge Society (EKS); 15(3): 149-171. Disponible en: https://revistas.usal.es/index. php/eks/article/view/12222

Rovira, I. (2018). Psicología y mente. Los 6 tipos de habilidades sociales, y para qué sirven. En: https://psicologiaymente.com/psicologia/ tipos-de-habilidades-sociales

Ruiz de Miguel, C. (2002). Factores familiares vinculados al bajo rendimiento. Revista Complutense de Educación. 12. En: http:// revistas.ucm.es/index.php/RCED/article/ viewFile/RCED0101120081A/16850

Ruiz, K. (2017). Engagement en Gerentes de Enfermería, Hospital Militar Escuela Dr. Alejandro Dávila Bolaños, Managua, 
Nicaragua. Mayo, Junio 2017. [Tesis master]. Managua-Nicaragua: Universidad Nacional Autónoma De Nicaragua, Managua. En: https://www.uv.es/seoane/boletin/previos/ N108-3.pdf

Rull,Á.(2019, octubre 23). Mejorartus habilidades sociales te ayudará a ser feliz. En El periodico website: https://www.elperiodico.com/es/serfeliz/20191023/mejorar-habilidades-socialesser-feliz-7687170

Salanova, M., Schaufeli, W., Llorens, S., Peiró, J. \& Grau, R. (2000). Desde el «burnout» al «engagement»: ¿Una nueva perspectiva? Revista de Psicología del Trabajo y de las Organizaciones, 16, 117-134.

Salanova, M. y Schaufeli, W.(2009). Elengagement en el trabajo. Cuando el trabajo se convierte en pasión. Madrid: Alianza Editorial.

Schaufeli, W. B., Martinez, I., Marques Pinto, A., Salanova, M., \& Bakker, A.B. (2002). Burnout and engagement in university students: Across national study. Journal of Cross- Cultural Psychology, 33, 464-481.

Schwartz, R. W. (1992). "Undergraduate surgical education for the twenty-first century", en Ann Surg, 216

Schwartz, R. W. (1994). "Assessing senior residents' knowledge and performance: an integrated evaluation program", en Surgery, 116.

Schuwirth, L. \& Van der Vleuten, C. (2004). "Different written assessment methods: what can be said about their strengths and weakness?", en $\mathrm{Med} E d u c, 38$.

88 Shih, S. (2012). An Examination of Academic Burnout Versus Work Engagement Among Taiwanese Adolescents. The Journal of Educational Research, 105, 286-298

Solano, L. (2015). Rendimiento académico de los estudiantes de secundaria obligatoria y su relación con las aptitudes mentales y las actitudes ante el estudio. (Tesis de posgrado). Universidad Nacional de Educación a Distancia, España.
Tapia, C., \& Cubo, S. (2017). Habilidades sociales relevantes: percepciones de múltiples actores educativos. Revista Internacional de Investigación en Educación, 133-148.

Van Der Vleuten, C. \& Schuwirth, L. (2005). "Assessing professional competence: from methods to programmes", en Med Educ, 39.

Wagner, M. F., Pereira, A. y Oliveira, M.S. (2014). Intervención sobre las dimensiones de la ansiedad social por medio de un programa de entrenamiento en habilidades sociales. Psicología Conductual, 22(3), 423-440.

Wass, V.(2001). "Composite undergraduate clinical examinations: how should the components be combined to maximize reliability?", en Med Educ, 35.

Willcox, M. del R. (2011). Factores de riesgo y protección para el rendimiento académico: Un estudio descriptivo en estudiantes de Psicología de una universidad privada. Revista Iberoamericana de Educación, 55(1), 1-9. En: http://www.rieoei.org/ deloslectores/3878Wilcox.pdf

Wilkinson, J., \& Canter, S. (2012). Social skills training manual: Assessment, programme design and management of training. Chichester: Wiley

Zavala, M., Valadez, M., \& Vargas, M. (2008). Inteligencia emocional y habilidades sociales en adolescentes con alta aceptación social. Revista Electrónica de Investigación Psicoeducativa, 319- 338.

Zúñiga F. (2017). Nivel de habilidades sociales en el adolescente de Iro de secundaria de la Institucion Educativa Franz Tomayo Solares 3096 Comas. (Tesis de grado) Universidad César Vallejo. En http://repositorio.ucv.edu.pe/bitstream/ handle/UCV/10916/Zu\%c3\%b1iga VA.pdf? sequence $=1 \&$ isAllowed $=y$ 